\title{
Uso de Práticas Integrativas e Complementares por idosos: Pesquisa Nacional de Saúde 2013
}

\author{
Use of Integrative and Complementary Practices by the elderly: \\ National Health Survey 2013
}

Priscila de Paula Marques ${ }^{\mathbf{1}}$, Priscila Maria Stolses Bergamo Francisco', Aldiane Gomes de Macedo Bacurau' ${ }^{\mathbf{1}}$ Patrícia Silveira Rodrigues ${ }^{\mathbf{1}}$ Deborah Carvalho Malta ${ }^{\mathbf{2}}$, Nelson Filice de Barros ${ }^{\mathbf{1}}$

DOI: $10.1590 / 0103-1104202012619$

RESUMO O estudo teve por objetivo estimar a prevalência da realização de Práticas Integrativas e Complementares (PIC) e sua relação com doenças crônicas em idosos brasileiros. Estudo transversal de base populacional realizado com dados da Pesquisa Nacional de Saúde (PNS/2013; n=23.815). Estimaramse a prevalência de realização de PIC e as frequências relativas das práticas referidas. Realizaram-se comparações entre proporções pelo teste de Rao-Scott com nível de significância de $5 \%$ e estimaram-se razões de prevalência para o uso das práticas integrativas e complementares, segundo doenças crônicas. $\mathrm{O}$ uso das PIC foi referido por 5,4\% (IC95\%:4,9-6,0) dos idosos. Entre estes, 62,6\% relataram uso de plantas medicinais/fitoterapia; $22,2 \%$, acupuntura; e 11,2\%, homeopatia. Somente $6,7 \%$ realizaram o tratamento no SUS. Observou-se maior realização das práticas pelas mulheres e para todos os tratamentos considerados $(\mathrm{p}<0,001)$; naqueles com colesterol alto, artrite ou reumatismo, problema de coluna e depressão $(\mathrm{p}<0,05)$. Os resultados dimensionam o uso das PIC com dados de abrangência nacional, apontando para sua utilização no tratamento das diversas condições de saúde que acometem principalmente os idosos.

PALAVRAS-CHAVE Terapias complementares. Prevalência. Saúde do idoso. Inquéritos de saúde. Doenças crônicas.

ABSTRACT The study aimed to estimate the prevalence of carrying out Integrative and Complementary Practices (PIC) and their relationship with chronic diseases in elderly Brazilians. Cross-sectional populationbased study conducted with data from the National Health Survey (PNS/2013; $n=23,815)$. The prevalence of carrying out PIC and the relative frequencies of the referred practices were estimated. Comparisons were made between proportions using the Rao-Scott test with a $5 \%$ significance level and prevalence ratios were estimated for the use of PIC, according to chronic diseases. The use of PIC was mentioned by 5.4\% (IC95\%: 4.9-6.0) of the elderly. Among these, 62.6\% reported the use of medicinal plants/phytotherapy; $22.2 \%$, acupuncture; and $11.2 \%$, homeopathy. Only $6.7 \%$ underwent treatment at Unified Health System (SUS). There was a greater performance of practices by women and for all treatments considered $(p<0.001)$; in those with high cholesterol, arthritis or rheumatism, spinal problems and depression $(p<0.05)$. The results measure the

1 Universidade Estadual de Campinas (Unicamp) Campinas (SP), Brasil. enf.primarques@gmail.com

2 Universidade Federal de Minas Gerais (UFMG)

- Belo Horizonte (MG), Brasil. use of integrative and complementary practices with national data, pointing to their use in the treatment of the various health conditions that mainly affect the elderly.

KEYWORDS Complementary therapies. Prevalence. Elderly health. Health surveys. Chronic diseases. 


\section{Introdução}

O envelhecimento é um processo natural e fisiológico da vida que, no Brasil, vem registrando crescimento acelerado e progressivo. Com a elevada prevalência de Doenças Crônicas Não Transmissíveis (DCNT), assim como das comorbidades a elas associadas e dos demais problemas de saúde que ocorrem com maior frequência nos idosos, os medicamentos ocupam papel central no tratamento e na recuperação, e constituem um dos itens mais importantes da atenção à saúde dos idosos ${ }^{1-5}$. Diante dessa realidade, torna-se maior a necessidade da adoção de ações de promoção de saúde e prevenção de agravos, principalmente no âmbito da atenção básica.

Práticas Integrativas e Complementares (PIC) é o termo usado no Brasil para designar a Medicina Tradicional (MT), a Medicina Alternativa e Complementar (MAC) e a Medicina Integrativa (MI) ${ }^{6}$ que, nas últimas décadas, têm recebido o estímulo da Organização Mundial da Saúde (OMS) para sua introdução na Atenção Primária à Saúde (APS) $)^{7-9}$. O uso das PIC ainda é pequeno, mas tem aumentado ${ }^{9-11}$, com variações entre as práticas e características sociodemográficas ${ }^{\mathbf{1 0}-12}$.

Embora o ritmo da institucionalização das PIC tenha sido lento', na APS, observa-se crescente revalorização das práticas não biomédicas, com ampliação da demanda, da legitimação social e da regulamentação institucional9,13. No Brasil, a Política Nacional de Práticas Integrativas e Complementares (PNPIC) foi institucionalizada no Sistema Único de Saúde (SUS) em 2006. Inicialmente, foram incorporados serviços de homeopatia, fitoterapia, acupuntura, antroposofia e termalismo. Em 2017, a PNPIC foi ampliada incluindo 14 outras práticas; e, em 2018, 10 novas práticas foram introduzidas ${ }^{\mathbf{1 4}}$.

A oferta desses recursos terapêuticos teve como foco principal a APS, proporcionando uma ampliação das abordagens de cuidado e das possibilidades terapêuticas aos usuários $^{\mathbf{1 4 , 1 5}}$. Dentre os diversos benefícios das PIC, destaca-se sua eficiência terapêutica, com viabilidade econômica para o sistema público de saúde ${ }^{16}$. As PIC são ofertadas em mais de 3 mil municípios, e $88 \%$ dessa oferta acontece em estabelecimentos da APS do SUS ${ }^{\mathbf{1 4}}$.

Dados sobre a prevalência de realização dessas práticas pela população idosa brasileira ainda são escassos na literatura. O objetivo do estudo foi estimar a prevalência da realização de PIC e sua relação com doenças crônicas em idosos brasileiros.

\section{Material e métodos}

Estudo quantitativo descritivo com dados de idosos que participaram da Pesquisa Nacional de Saúde (PNS), realizada em 2013 pelo Ministério da Saúde em parceria com o Instituto Brasileiro de Geografia e Estatística (IBGE), e que coletou informações sobre diversos aspectos relacionados com a saúde da população brasileira.

A PNS utilizou amostragem por conglomerados em três estágios de seleção, tendo como unidade primária de amostragem os setores censitários ou conjuntos de setores, selecionados por amostragem aleatória simples. Os domicílios particulares permanentes constituíram as unidades secundárias; e, em cada domicílio, foi selecionado, por amostragem aleatória simples, um morador adulto (com idade $\geq 18$ anos), para compor o conjunto das unidades no terceiro estágio. Detalhes sobre o processo de amostragem e ponderação estão disponíveis em publicações prévias ${ }^{\mathbf{1 7 1 8}}$.

O instrumento de coleta de dados utilizado na PNS se dividiu em três partes: os questionários domiciliar e sobre todos os moradores (respondidos por um residente que sabia informar sobre a situação socioeconômica e de saúde de todos - informante proxy), e o questionário individual (respondido exclusivamente pelo morador com idade $\geq 18$ anos, selecionado com equiprobabilidade entre todos os residentes no domicílio). Os adultos com 60 anos ou mais foram incluídos na pesquisa 
e responderam ao módulo específico sobre a saúde do idoso ${ }^{17-20}$. Cabe destacar que, quando o residente não estava presente ou não estava em condições de responder, o questionário foi respondido pelo responsável do domicílio, ou o proxy ${ }^{20}$.

Para o presente estudo, foram considerados todos os participantes com 60 anos ou mais de idade ( $\mathrm{n}=23.815$ ), para a análise dos dados relativos às PIC do bloco sobre utilização de serviços de saúde, que incluem os próprios respondentes e informações referidas pelo morador proxy. As perguntas foram: (J53) 'Nos últimos 12 meses, __ utilizou alguma prática integrativa e complementar, isto é, tratamento como acupuntura, homeopatia, plantas medicinais e fitoterapia etc.?' (sim ou não). Para os que responderam positivamente (n=1.420): (J54) - 'Qual tratamento _ _ fez uso? (acupuntura, homeopatia, plantas medicinais e fitoterapia, outro)'; e (J57) - 'Este tratamento foi feito através do Sistema Único de Saúde (SUS)?' (sim ou não).

Também, foram consideradas algumas DCNT referidas relativas ao próprio morador selecionado na mesma faixa etária $(\mathrm{n}=11.177)$, quais sejam: hipertensão arterial; diabetes; colesterol alto; doença do coração; Acidente Vascular Cerebral (AVC) ou derrame; asma (ou bronquite asmática); artrite ou reumatismo; câncer; insuficiência renal crônica; problema crônico de coluna, como dor crônica nas costas ou no pescoço, lombalgia, dor ciática, problemas nas vértebras ou disco; depressão e doença no pulmão (enfisema pulmonar, bronquite crônica ou Doença Pulmonar Obstrutiva Crônica - DPOC). As informações se referem ao relato do diagnóstico médico prévio, na maioria das DCNT, exceto para dor crônica de coluna (autodeclarada) e depressão que considerou diagnóstico prévio de médico ou outro profissional de saúde mental (psiquiatra ou psicólogo). Todas as doenças crônicas foram categorizadas em 'sim' ou 'não'. Essas respostas foram prestadas pelo próprio morador com 60 anos ou mais que respondeu o questionário individual.

Os microdados da PNS encontram-se disponíveis para uso público no site https:// ww2.ibge.gov.br/home/estatistica/populacao/ pns/2013_vol4. Estimaram-se a prevalência de realização das práticas e respectivos intervalos de $95 \%$ de confiança (IC95\%) e, para as demais variáveis relativas às PIC, verificou-se a distribuição percentual pelas frequências relativas pontuais e por intervalo (ponderadas). Foram comparadas as frequências de uso das PIC segundo o sexo pelo teste Qui-Quadrado de Pearson (Rao-Scott), considerando-se um nível de significância de 5\%; estimaram-se as prevalências do uso de PIC segundo as DCNT e as razões de prevalência ajustadas por sexo e idade pela regressão de Poisson.

Todas as análises foram realizadas por meio do módulo survey no Stata 14.0 (StataCorp LP, College Station, Estados Unidos), considerando-se o desenho complexo de amostragem da pesquisa. A PNS 2013 foi aprovada pela Comissão Nacional de Ética em Pesquisa para Seres Humanos do Ministério da Saúde (Parecer $\mathrm{n}^{\circ} 328.159$, de 26 de junho de 2013).

\section{Resultados}

A média de idade dos idosos foi de 69,9 anos (IC95\%:69,7-70,1); e 56,4\% eram mulheres. O uso de PIC foi referido por 5,4\% (IC95\%:4,9$6,0)$ dos idosos, e a distribuição percentual dos tipos de PIC realizadas é apresentada no gráfico 1. A maioria relatou o uso de plantas medicinais e fitoterapia (62,6\%; IC95\%:59,7-65,5), seguida por acupuntura $(22,2 \%$; IC95\%:20,2$24,4)$ e homeopatia (11,2\%; IC95\%:8,9-13,9). 
Gráfico 1. Distribuição percentual das Práticas Integrativas e Complementares (PIC) realizadas pelos idosos nos últimos 12 meses $(n=23.815)$. Pesquisa Nacional de Saúde, 2013

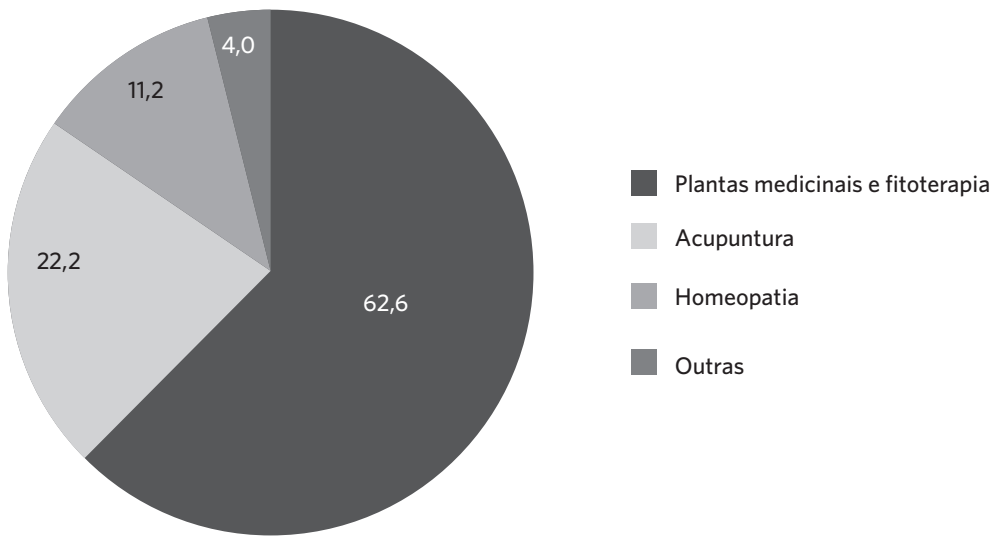

Fonte: Elaboração própria.

Somente 6,7\% (IC95\%:5,1-8,7) dos idosos referiram a realização do tratamento em serviços do SUS à época da pesquisa. Observaram-se diferenças estatísticas significativas nas prevalências de realização das práticas entre os sexos $(4,3 \% \mathrm{e}$ 6,3\% em homens e mulheres respectivamente), assim como para todos os tipos de tratamentos considerados $(\mathrm{p}<0,001)$ (gráfico 2).

Gráfico 2. Tratamentos realizados pelos idosos nos últimos 12 meses, segundo sexo $(n=23.815)$. Pesquisa Nacional de Saúde, 2013

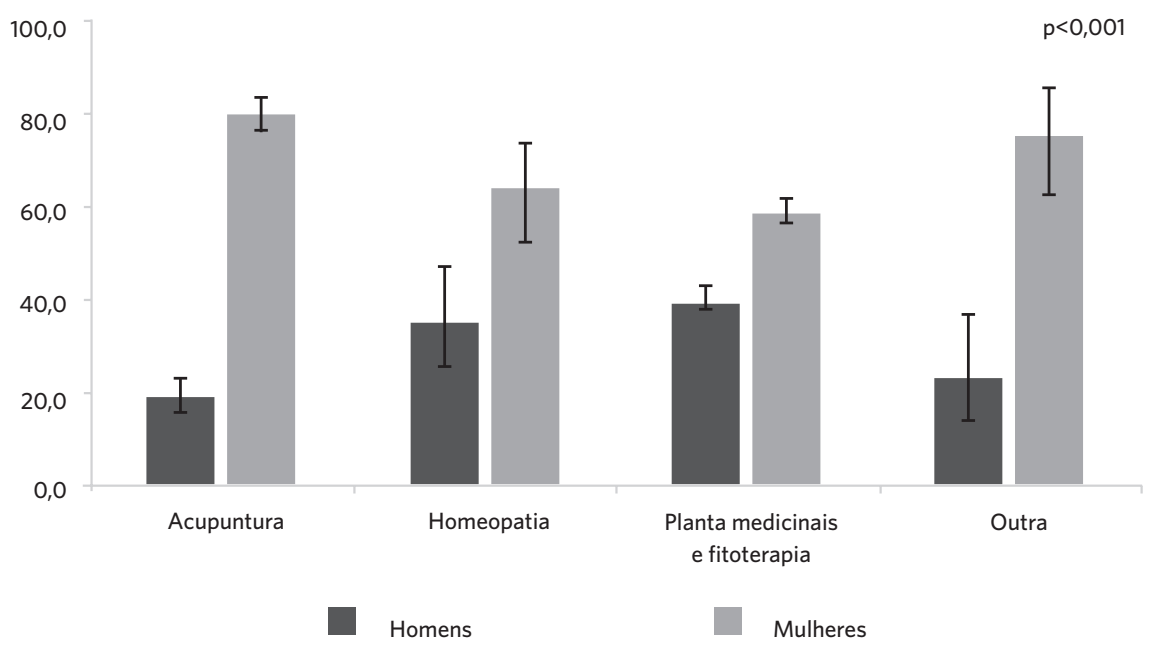


Quanto ao uso de práticas alternativas e complementares, de acordo com a presença de doenças crônicas, foram verificadas, após ajuste por sexo e idade, maiores proporções de uso de PIC entre os idosos com colesterol alto (RP=1,42; IC95\%: 1,10-1,83), artrite ou reumatismo ( $\mathrm{RP}=1,75$; IC95\%: 1,35-2,27); problema crônico de coluna ( $\mathrm{RP}=1,89$; IC95\%: 1,50-2,38) e depressão ( $\mathrm{RP}=1,62$; IC95\%: 1,18-2,21) (tabela 1).

Tabela 1. Prevalência e razão de prevalência do uso de práticas alternativas e complementares, de acordo com a presença de doenças crônicas em idosos brasileiros (n=11.177). Pesquisa Nacional de Saúde, 2013

\begin{tabular}{|c|c|c|c|c|c|}
\hline Doenças crônicas & $\mathrm{n}$ & $\%$ & IC95\% & Valor $\mathrm{p}$ & RP (IC95\%) \\
\hline Hipertensão arterial & 5.524 & 5,1 & $4,3-5,9$ & 0,352 & $0,87(0,69-1,09)$ \\
\hline Diabetes & 1.896 & 4,9 & $3,8-6,3$ & 0,447 & $0,88(0,67-1,16)$ \\
\hline Colesterol alto & 2.604 & 7,2 & $5,8-8,9$ & 0,002 & $1,42(1,10-1,83)$ \\
\hline Doença do coração & 1.122 & 5,5 & $3,7-8,0$ & 0,941 & $1,03(0,69-1,55)$ \\
\hline $\mathrm{AVC}^{\star}$ ou derrame & 563 & 5,8 & $3,6-9,2$ & 0,761 & $1,13(0,69-1,85)$ \\
\hline Asma (ou bronquite asmática) & 495 & 4,5 & $3,0-6,8$ & 0,421 & $0,82(0,53-1,27)$ \\
\hline Artrite ou reumatismo & 1.880 & 8,7 & $7,0-10,8$ & $<0,001$ & $1,75(1,35-2,27)$ \\
\hline $\begin{array}{l}\text { Problema crônico de coluna, como } \\
\text { dor crônica nas costas ou no pescoço, } \\
\text { lombalgia, dor ciática, problemas nas } \\
\text { vértebras ou disco }\end{array}$ & 2.897 & 8,2 & $6,8-10,0$ & $<0,001$ & $1,89(1,50-2,38)$ \\
\hline Depressão & 953 & 8,8 & $6,5-11,6$ & $<0,001$ & $1,62(1,18-2,21)$ \\
\hline $\begin{array}{l}\text { Doença no pulmão ou DPOC }{ }^{\star \star} \text {, tais } \\
\text { como enfisema pulmonar, bronquite } \\
\text { crônica ou outro }\end{array}$ & 352 & 4,5 & $2,3-8,8$ & 0,607 & $0,85(0,42-1,70)$ \\
\hline Câncer & 542 & 7,2 & $4,8-10,7$ & 0,146 & $1,44(0,94-2,20)$ \\
\hline Insuficiência renal crônica & 283 & 8,8 & $5,1-14,7$ & 0,066 & $1,72(1,00-2,97)$ \\
\hline
\end{tabular}

Fonte: Elaboração própria.

*AVC: Acidente Vascular Cerebral; **DPOC: Doença Pulmonar Obstrutiva Crônica.

\section{Discussão}

Os resultados revelaram baixa prevalência de uso das PIC pelos idosos. Para todas as práticas consideradas, observou-se maior proporção entre mulheres; e, quanto ao serviço, pequeno percentual referiu tratamento pelo SUS à época da pesquisa. O maior uso das PIC pelas mulheres tem sido observado em outros estudos ${ }^{12,21}$. As práticas constituem uma alternativa necessária de abordagem terapêutica ao cuidado do idoso, e o Brasil é um dos poucos países no mundo a ofertar as PIC de forma gratuita, por meio do SUS, em todos os níveis de atenção $0^{6,14,15}$.

Neste estudo, observaram-se associações positivas entre o uso das PIC e a presença de hipercolesterolemia, artrite ou reumatismo, problema de coluna e depressão. Estudo realizado com dados de 2012 do National Health Interview Survey e do Adult Alternative Medicine para verificar o uso das práticas 
entre pessoas com múltiplas condições crônicas ( $\mathrm{n}=15.463)$ e que incluiu idosos verificou que os participantes com hipercolesterolemia apresentaram maiores proporções de uso das práticas quando comparados àqueles sem essa condição $(p<0,001)^{21}$. No presente estudo, o uso das práticas foi $42 \%$ maior nos idosos que referiram colesterol alto.

O uso das PIC é elevado entre indivíduos com doenças reumáticas ${ }^{22-24}$, geralmente concomitante ao tratamento médico convencional e com avaliação positiva quanto aos efeitos ${ }^{23}$. Neste estudo, verificou-se maior uso das práticas entre os idosos com artrite ou reumatismo $(\mathrm{RP}=1,75)$. Ressalta-se a necessidade de que os profissionais de saúde que acompanham os portadores dessas condições crônicas sejam informados sobre o uso concomitante de ambos os tratamentos, e considerem suas potencialidades no cuidado.

Diversos estudos evidenciam os benefícios das PIC ${ }^{25-31}$, incluindo o uso de plantas

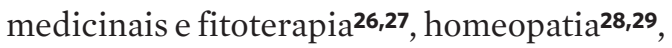
acupuntura em morbidades prevalentes nos idosos ${ }^{30,31}$, bem como a redução do uso de medicamentos ${ }^{30}$. Neste estudo, observou-se maior prevalência de uso das práticas nos idosos que relataram problema crônico de coluna - como dor nas costas, lombalgia, entre outros. Revisão sistemática com 13 estudos longitudinais sobre o uso da acupuntura no tratamento de dor crônica demonstrou resultados satisfatórios quando comparado aos tratamentos tradicionais, ou nenhum tratamento para dor lombar, osteoartrite e dor de cabeça. Também foi verificada redução do uso de analgésicos ${ }^{30}$.

Nos idosos com depressão, observou-se que o uso das práticas foi $62 \%$ maior, independentemente do sexo e da idade. Revisão bibliográfica apontou a efetividade do uso do Ginkgo biloba L. (Ginkgo) na insuficiência cerebral e tratamento de demência (Alzheimer), bem como para o Hypericum perforatum (Hipérico) no tratamento de depressão leve a moderada ${ }^{27}$. Ensaio clínico realizado no México para avaliar o tratamento homeopático individualizado e fluoxetina para depressão moderada a grave em mulheres na peri e pós-menopausa (idade entre 40 a 65 anos) evidenciou, após seis semanas de tratamento, a efetividade da homeopatia no tratamento para depressão, inclusive em mulheres na menopausa com estados depressivos de moderado a grave; a homeopatia, mas não a fluoxetina, melhorou os sintomas da menopausa avaliados pela Greene Climacteric Scale ${ }^{28}$.

Entre as condições crônicas que acometem os idosos, a hipertensão arterial é a mais prevalente. Neste estudo, não foi observada relação entre hipertensão e o uso das PIC, diferentemente do que verificou Mbizo et al. ${ }^{21}$ em estudo com adultos e idosos americanos. Resultados de ensaio clínico sobre o uso de acupuntura realizado com pacientes em emergências hipertensivas na APS ( $\mathrm{n}=108)$ mostraram que, no grupo que recebeu a intervenção terapêutica, houve melhora dos níveis pressóricos em $98 \%$ dos casos ${ }^{31}$. Estudo observacional prospectivo sobre tratamento homeopático de pacientes com idade $>70$ anos $(n=83)$, acompanhados por dois anos e cujos diagnósticos mais frequentes foram hipertensão arterial e distúrbios do sono, apontou redução marcante e sustentada da gravidade das queixas ${ }^{29}$.

No Brasil, como parte do cuidado integral aos portadores de doenças crônicas, observou-se a ampliação do acesso aos medicamentos nos últimos anos ${ }^{32}$. O tratamento medicamentoso é necessário para o controle das doenças crônicas, redução da morbimortalidade e melhoria da qualidade de vida dos usuários ${ }^{4,32}$. O SUS, por meio da assistência farmacêutica, deve garantir o acesso, mas, principalmente, promover o uso racional dos medicamentos ${ }^{33,34}$. Os benefícios e a recomendação das PIC, especialmente para os idosos, são estratégias que podem também contribuir para a redução dos problemas relacionados com o uso de medicamentos nessa faixa etária ${ }^{35}$.

Neste estudo, principalmente, o uso de plantas medicinais e de fitoterápicos foi mencionado pelos idosos, decorrente de 
conhecimentos empíricos acumulados pela população brasileira sobre seus efeitos para o cuidado da saúde. Cabe ressaltar que, mesmo diante da existência de uma política nacional específica para o uso de plantas medicinal e fitoterápico, alinhado à PNPIC, dos medicamentos constarem na Relação Nacional de Medicamentos Essenciais (Rename) vigente e de possuírem financiamento assegurado por meio do Componente Básico da Assistência Farmacêutica (CBAF), menos de 3\% dos municípios disponibilizavam medicamentos fitoterápicos à população ${ }^{36,37}$. Ressalta-se que os profissionais de saúde ainda se sentem despreparados para a prescrição de fitoterápicos ${ }^{37}$ e para orientações sobre o uso seguro e eficaz de plantas medicinais, situação essa também verificada para as demais PIC $^{\mathbf{3 8}}$. Porém, a PIC pode ser utilizada para integração interprofissional nas equipes de atenção primária, promoção da saúde e estratégia de aproximação entre profissionais e usuários ${ }^{39}$.

Com o envelhecimento da população brasileira, torna-se necessário maior estímulo para o uso do conjunto das PIC ofertadas pelo SUS que, para além do tratamento, também promovem o autoconhecimento e a autonomia pessoal, auxiliando na redução dos danos à saúde causados pelos impactos sociais vividos pelos idosos, e ressignificando, nessa fase da vida, o bem-estar físico e mental15, para que esses anos sejam vividos com qualidade e dignidade.

$\mathrm{Na}$ atenção à saúde do idoso, a prestação de serviços fragmenta a atenção, com ampliação de consultas a especialistas, informações não compartilhadas, uso excessivo de fármacos, exames clínicos e de imagem, e vários outros procedimentos que sobrecarregam o sistema, com forte impacto financeiro em todos os níveis; e não geram, necessariamente, benefícios significativos para a saúde do idoso e para a sua qualidade de vida ${ }^{40}$. No entanto, é importante considerar que a busca por PIC para o tratamento e a recuperação da saúde pode ser uma forma de afirmação de uma identidade de cuidado oposta às práticas de cuidado convencionais ${ }^{41}$, além de apontar uma valorização de práticas já conhecidas tradicionalmente pelas populações e, muitas vezes, não valorizadas. Destacam-se as barreiras comunicação médico-paciente quanto ao uso das PIC observadas também em outros países ${ }^{\mathbf{4 2}, 43}$.

Este estudo dimensionou o uso de PIC em idosos brasileiros que, necessariamente, relaciona-se com a oferta e acesso ao serviço. Devem-se considerar as diversidades regionais, estaduais e municipais quanto à implementação das PIC no País. Estudo utilizando dados do segundo ciclo da avaliação externa do Programa Nacional de Melhoria do Acesso e da Qualidade da Atenção Básica (PMAQ$\mathrm{AB} / 2013$ ) com amostra que contemplou 1.470 equipes, que atendem a 293 municípios de Santa Catarina (SC), observou resultado de implantação expressivo já que maioria da população do estado tinha acesso às PIC. Verificaram $56,3 \%$ do estado com PIC implantadas ou parcialmente implantadas, com diferenças entre municípios com portes populacionais diver$\operatorname{sos}^{\mathbf{4 4}}$. Os autores consideram que a ampliação e a implantação das PIC na atenção básica, 'a gestão federal deva assumir de fato a PNPIC como uma política de Estado, garantindo recursos financeiros para sua atuação no SUS e permitindo que o esforço das equipes que ofertam PIC transformem-se em experiências mais exitosas, não permanecendo apenas uma política focal e isolada'.

Entre as limitações do estudo, deve-se considerar as questões inerentes à natureza transversal da pesquisa, que torna impossível definir uma relação de causalidade para as associações identificadas, como o uso de práticas alternativas e complementares e algumas doenças crônicas. Todas as informações foram obtidas por meio de autorrelato, algumas delas respondidas por um informante proxy (substituto) ${ }^{\mathbf{1 7}, \mathbf{1 8}}$. No entanto, ressalta-se o uso de dados obtidos de pesquisa de base populacional representativa da população adulta brasileira, que permitiu estimativas inéditas da prevalência do uso das PIC entre os idosos. 
Para superar os desafios da assistência integral à saúde dos idosos, é imperativa a reorganização de processos de trabalhos nas redes de atenção e cuidado à saúde $\mathbf{4 0 , 4 5}^{\text {. Para }}$ Cecílio \& Merhy:

Uma complexa trama de atos, de procedimentos, de fluxos, de rotinas, de saberes, num processo dialético de complementação, mas também de disputa, compõe o que entendemos como cuidado em saúde46(200).

Deve-se considerar que a integralidade da atenção é resultante das formas como se articulam as diferentes práticas dos trabalhadores no cuidado à saúde do idoso.

\section{Considerações finais}

Os resultados revelaram baixa prevalência de uso das PIC entre os idosos, porém, maior entre as mulheres. Naqueles com colesterol alto, artrite ou reumatismo, problema crônico de coluna e depressão, observou-se maior proporção de realização de PIC. Na atenção à saúde do idoso, de modo geral, outras formas de cuidado terapêutico, além do tratamento medicamentoso, ainda são secundárias na prática médica. Os dados da PNS revelaram pequena utilização desses recursos não biomédicos específicos - disponíveis no SUS em 2013 - no tratamento das diversas condições de saúde que acometem os idosos, tanto das práticas baseadas em avaliações científicas de eficácia e segurança (integrativas) quanto daquelas usadas de forma paralela (complementares) à medicina convencional.

\section{Agradecimentos}

Agradecemos ao Ministério da Saúde e ao IBGE pela disponibilização dos dados da PNS 2013.

\section{Colaboradores}

Marques PP (0000-0002-7954-4056)* contribuiu para concepção do estudo, revisão da literatura e redação do manuscrito. Francisco PMSB (0000-0001-7361-9961)* contribuiu para concepção do estudo, análise e interpretação dos dados e redação do manuscrito. Bacurau AGM (0000-0002-6671-2284)* colaborou para análise e interpretação dos dados e redação do manuscrito. Rodrigues PS (0000-0002-1313-2403)* contribuiu para a revisão da literatura e redação do manuscrito. Malta DC (0000-0002-8214-5734)* contribuiu para a revisão crítica do conteúdo. Barros NF (0000-0002-5404-1516)* contribuiu para a revisão da literatura e para a revisão crítica do conteúdo. Todos os autores aprovaram a versão final do manuscrito. 


\section{Referências}

1. Pereira CG, Peres MA, Iop D, et al. Polifarmácia em idosos: um estudo de base populacional. Rev. Bras. Epidemiol. [internet]. 2017 [acesso em 2019 ago 27]; 20(2):335-44. Disponível em: http://dx.doi. org/10.1590/1980-5497201700020013.

2. Ramos LR, Tavares NUL, Bertoldi AD, et al. Polypharmacy and Polymorbidity in Older Adults in Brazil: a public health challenge. Rev. Saude Publica [internet]. 2016 [acesso em 2019 ago 27]; 50(suppl2):9s. Disponível em: http://dx.doi.org/10.1590/s15188787.2016050006145.

3. Tavares NUL, Costa KS, Mengue SS, et al. Brasil: resultados da Pesquisa Nacional de Saúde, 2013. Epidemiol. Serv. Saude [internet]. 2015 [acesso em 2019 ago 27]; 24(2):315-23. Disponível em: https://doi.org/10.5123/ S1679-49742015000200014.

4. Lulita MF, Girouard H. Treating hypertension to prevent cognitive decline and dementia: re-opening the debate. Adv. Exp. Med. Biol. [internet]. 2017 [acesso em 2020 maio 6]; (956):447-73. Disponível em: https://doi.org/10.1007/5584_2016_98.

5. Romano-Lieber NS, Corona LP, Marques LFG, et al. Sobrevida de idosos e exposição à polifarmácia no município de São Paulo: Estudo SABE. Rev. Bras. Epidemiol. [internet]. 2018 [acesso em 2020 maio 6]; 21(supl2):E180006. Disponível em: https://doi. org/10.1590/1980-549720180006.supl.2.

6. Brasil. Ministério da Saúde. Portaria n ${ }^{\circ}$ 971, de 4 de maio de 2006. Dispõe sobre a Política Nacional de Práticas Integrativas e Complementares (PNPIC) no Sistema Único de Saúde. Diário Oficial da União. 5 Maio 2006.

7. Organización Mundial de la Salud. Estratégia de la OMS sobre medicina tradicional 2002-2005. Genebra: OMS; 2001.

8. World Health Organization. WHO Global Report on Traditional and Complementary Medicine 2019. Geneva: World Health Organization; 2019.
9. World Health Organization. WHO traditional medicine strategy: 2014-2023. Geneva: WHO; 2013.

10. Clarke TC, Black LI, Stussman BJ, et al. Trends in the Use of Complementary Health Approaches Among Adults: United States, 2002-2012. Natl. Health. Stat. Report. 2015; (79):1-16.

11. Reid R, Steel A, Wardle J, et al. Complementary medicine use by the Australian population: a critical mixed studies systematic review of utilisation, perceptions and factors associated with use. BMC Complement. Altern. Med. [internet]. 2016 [acesso em 2019 ago 27]; 16(176). Disponível em: https://dx.doi. org/10.1186/s12906-016-1143-8.

12. Zang $\mathrm{Y}$, Leach MJ, Hall H, et al. Differences between Male and Female Consumers of Complementary and Alternative Medicine in a National US Population: A Secondary Analysis of 2012 NIHS Data. Evid. Based Complement. Altern. Med. [internet]. 2015 [acesso em 2019 ago 27]; (413173):1-11. Disponível em: https:// dx.doi.org/10.1155/2015/413173.

13. Tesser CD, Sousa IMC, Nascimento MC. Práticas Integrativas e Complementares na Atenção Primária à Saúde brasileira. Saude debate [internet]. 2018 [acesso em 2019 ago 27]; 42(esp1):174-88. Disponível em: http://dx.doi.org/10.1590/0103-11042018s112.

14. Brasil. Ministério da Saúde. Liderança na Atenção Básica. Saúde consolida 29 modalidades de práticas integrativas no SUS. Brasília, DF: MS; 2018.

15. Brasil. Ministério da Saúde. Práticas integrativas e complementares: plantas medicinais e fitoterapia na Atenção Básica. Brasília, DF: MS; 2012.

16. Fischborn AF, Machado J, Fagundes NC, et al. A Política das práticas integrativas e complementares do SUS: o relato de experiência sobre a implementação em uma unidade de ensino e serviço de saúde. Cinergis [internet]. 2016. [acesso em 2019 ago 27]; 17(4supl1):358-63. Disponível em: http://dx.doi. org/10.17058/cinergis.v17i0.8149. 
17. Souza-Júnior PRB, Freitas MPS, Antonaci GA, et al. Sampling design for the National Health Survey, 2013. Epidemiol. Serv. Saude. [internet]. 2015 [acesso em 2019 ago 27]; 24(2):207-16. Disponível em: http://www.scielo.br/pdf/ress/v24n2/2237-9622ress-24-02-00207.pdf.

18. Szwarcwald CL, Malta DC, Pereira CA, et al. Pesquisa Nacional de Saúde no Brasil: concepção e metodologia de aplicação. Ciênc. Saúde Colet. [internet]. 2014. [acesso em 2019 ago 27]; 19(2):333-42. Disponível em: http://dx.doi.org/10.1590/1413-81232014192.14072012.

19. Oliveira-Figueiredo DST, Felisbino-Mendes MS, Malta DC, et al. Prevalência de incapacidade funcional em idosos: análise da Pesquisa Nacional de Saúde. Rev. Rene [internet]. 2017 [acesso em 2019 ago 27]; 18(4):468-75. Disponível em: http://dx.doi. org/10.15253/2175-6783.2017000400007.

20. Instituto Brasileiro de Geografia e Estatística. Coordenação de Trabalho e Rendimento. Pesquisa Nacional de Saúde 2013: Ciclos de vida: Brasil e grandes regiões. Rio de Janeiro: IBGE; 2013.

21. Mbizo J, Okafor A, Sutton MA, et al. Complementary and alternative medicine use among persons with multiple chronic conditions: results from the $2012 \mathrm{Na}-$ tional Health Interview Survey. BMC Complement. Alter. Med. [internet] 2018 [acesso em 2020 maio 6]; 18(1):281. Disponível em: https://doi.org/10.1186/ s12906-018-2342-2.

22. Yang S, Dubé CE, Eaton CB, et al. Longitudinal use of complementary and alternative medicine among older adults with radiographic knee osteoarthritis. Clin Ther [internet] 2013 [acesso em 2020 maio 6]; 35(11):1690-702. Disponível em: https://doi. org/10.1016/j.clinthera.2013.09.022.

23. Yang L, Sibbritt D, Adams J. A critical review of complementary and alternative medicine use among people with arthritis: a focus upon prevalence, cost, user profiles, motivation, decision-making, perceived benefits and communication. Rheumatol. Int. [internet] 2017 [acesso em 2020 maio 6]; 37(3):337-51. Disponível em: https://doi.org/10.1007/s00296-016-3616-y.
24. Drieskens S, Tafforeau J, Demarest S. Do sociodemographic characteristics associated with the use of CAM differ by chronic disease? Eur. J. Public Health. 2019; 29(4):655-60.

25. Dacal MPO, Silva IS. Impacto das práticas integrativas e complementares na saúde de pacientes crônicos. Saúde debate. 2018; 42(118):724-35.

26. Alexandre RFA, Garcia FN, Simões CMO. Fitoterapia baseada em evidências. Parte 2. Medicamentos fitoterápicos elaborados com alcachofra, castanha-da-índia, ginseng e maracujá. Acta Farm. Bonaerense. 2005; 24(2):310-14.

27. Alexandre RFA, Garcia FN, Simões CMO. Fitoterapia baseada em evidências. Parte 1. Medicamentos fitoterápicos elaborados com ginkgo, hipérico, kava e valeriana. Acta Farm. Bonaerense. 2005; 24(2):300-09.

28. Macías-Cortés EC, Llanes-González L, Aguilar-Faisal L, et al. Individualized homeopathic treatment and fluoxetine for moderate to severe depression in peri-and postmenopausal women (HOMDEP-MENOP study): a randomized, double-dummy, double-blind, placebo-controlled trial. PLoS One [internet]. 2015 [acesso em 2019 ago 28]; 10(3):e0118440. Disponível em: https://www.ncbi.nlm.nih.gov/pmc/articles/PMC4359147/.

29. Teut M, Lüdtke R, Schnabel K, et al. Homeopathic treatment of elderly patients-a prospective observational study with follow-up over a two year period. BMC Geriatr. [internet]. 2010 [acesso em 2019 ago 28]; 10(1):10. Disponível em: http://dx.doi.org/10.1186/1471-231810-10.

30. Dalamagka M. Systematic Review: Acupuncture in Chronic Pain, Low Back Pain and Migraine. J Pain Relief [internet]. 2015 [acesso em 2019 ago 28]; 4(5):000195. Disponível em: http://dx.doi. org/10.4172/2167-0846.1000195.

31. Chaveco BG, Mederos AME, Vaillant OS, et al. Eficacia del tratamiento acupuntural en pacientes com urgencias hipertensivas en la atención primaria de salud. Medisan. 2011; 15(11):1557-65. 
32. Malta DC, Silva Junior JB. O plano de ações estratégicas para o enfrentamento das doenças crônicas não transmissíveis no Brasil e a definição das metas globais para o enfrentamento dessas doenças até 2025: uma revisão. Epidemiol. Serv. Saude [internet]. 2013 [acesso em 2019 ago 27]; 22(1):151-64. Disponível em: http://dx.doi.org/10.5123/S1679-49742013000100016.

33. Akerman M, Freitas O. Pesquisa Nacional sobre acesso, utilização e promoção do uso racional de medicamentos (PNAUM): avaliação dos serviços de atenção farmacêutica primária. Rev. Saude Publica [internet] 2017 [acesso em 2020 maio 6]; 51(supl2):1s. Disponível em: https://doi.org/10.11606/S1518-8787.201705100supl2ed.

34. Costa KS, Tavares NUL, Junior JMN, et al. Avanços e desafios da assitência farmacêutica na atenção primária no Sistema Único de Saúde. Rev. Saude Publica [internet] 2017 [acesso em 2020 maio 6]; 51(supl2:3s). Disponível em: https://doi.org/10.11606/ S1518-8787.2017051007146.

35. Novaes PH, Cruz DT, Lucchetti ALG, et al. The "iatrogenic triad": Polypharmacy, drug-drug interactions, and potentially inappropriate medications in older adults. Int. J. Clin. Pharm. [internet]. 2017 [acesso em 2019 ago 28]; 39:818-25. Disponível em: http:// dx.doi.org/10.1007/s11096-017-0470-2.

36. Brasil. Ministério da Saúde. Portaria $n^{\circ} 1.555$, de 30 de julho de 2013. Dispõe sobre as normas de financiamento e de execução do Componente Básico da Assistência Farmacêutica no âmbito do Sistema Único de Saúde (SUS). Diário Oficial da União. 31 Jul 2013.

37. Brasil. Ministério da Saúde. Portaria n ${ }^{\circ}$ 2.077, de 17 de setembro de 2012. Institui a Pesquisa Nacional sobre Acesso, Utilização e Promoção do Uso Racional de Medicamentos no Brasil (PNAUM). Diário Oficial da União. 18 Set 2012.

38. Nascimento Junior BJ, Tínel LO, Silva ES, et al. Avaliação do conhecimento e percepção dos profissionais da estratégia de saúde da família sobre o uso de plantas medicinais e fitoterapia em Petrolina-PE, Bra- sil. Rev. Bras. Plantas Med. [internet]. 2016 [acesso em 2019 ago 28]; 18(1):57-66. Disponível em: http:// dx.doi.org/10.1590/1983-084X/15_031.

39. Barros NF, Spadacio C, Costa MV. Trabalho interprofissional e as Práticas Integrativas e Complementares no contexto da Atenção Primária à Saúde: potenciais e desafios. Saúde debate [internet] 2018 [acesso em 2020 maio 6]; 42(espl):163-73. Disponível em: https://doi.org/10.1590/0103-11042018s111.

40. Oliveira MR, Veras RP, Cordeiro HA. A importânica da porta de entrada no sistema: o modelo integral de cuidado para o idoso. Physis [internet] 2018 [acesso em 2020 maio 6]; 28(4):e280411. Disponível em: https://doi.org/10.1590/S0103-73312018280411.

41. Telesi Júnior E. Práticas integrativas e complementares em saúde, uma nova eficácia para o SUS. Estud. Av. [internet]. 2016 [acesso em 2019 ago 28]; 30(86):99112. Disponível em: http://dx.doi.org/10.1590/S010340142016.00100007.

42. Shelley BM, Sussman AL, Williams RL, et al. They don't ask me so I don't tell them: Patient-clinician communication about traditional, complementary, and alternative medicine. Ann. Fam. Med. [internet]. 2009 [acesso em 2019 ago 28]; 7(2):139-47. Disponível em: http://dx.doi.org/10.1370/afm.947.

43. Jou J, Johnson PJ. Nondisclosure of Complementary and Alternative Medicine Use to Primary Care Physicians: Findings From the 2012 National Health Interview Survey. JAMA Intern. Med. [internet]. 2016 [acesso em 2019 ago 28]; 176(4):545-46. Disponível em: http://dx.doi.org/10.1001/jamainternmed.2015.8593.

44. Losso LN, Freitas SFT. Avaliação do grau da implantação das práticas integrativas e complementares na Atenção Básica em Santa Catarina, Brasil. Saúde debate [internet]. 2017 [acesso em 2019 ago 28]; 41(3):17187. Disponível em: http://dx.doi.org/10.1590/0103$-11042017 \mathrm{~s} 313$.

45. Veras RP, Oliveira M. Envelher no Brasil: a constru- 
ção de um modelo de cuidado. Ciênc. Saude Colet. [internet] 2018 [acesso em 2020 maio 7]; 23(6):192936. Disponível em: http://dx.doi.org/10.1590/141381232018236.04722018 .

46. Cecílio LCO, Merhy EE. A integralidade do cuidado como eixo da gestão hospitalar. In: Pinheiro R, Mat- tos RA, organizadores. Construção da Integralidade: cotidiano saberes e práticas em saúde. Rio de Janeiro: IMS; Abrasco; 2003. p. 197-210.

Recebido em 25/09/2019

Aprovado em 14/05/2020

Conflito de interesses: inexistente

Suporte financeiro: não houve 